\title{
Healthy living in pregnancy: a cluster-randomized controlled trial to prevent excessive gestational weight gain - rationale and design of the GeliS study
}

Kathrin Rauh ${ }^{1,2+}$, Julia Kunath ${ }^{1,3+}$, Eva Rosenfeld ${ }^{2}$, Luzia Kick², Kurt Ulm ${ }^{4}$ and Hans Hauner ${ }^{1,3 *}$

\begin{abstract}
Background: Recent studies suggest that excessive gestational weight gain (GWG) leads to adverse maternal and fetal outcomes including weight retention in the mother and an increased risk of childhood obesity in the offspring.

The aim of the GeliS study is to examine the effect of a lifestyle intervention programme during pregnancy to avoid excessive GWG and, hence, to reduce pregnancy and obstetric complications as well as the risk of maternal and offspring obesity.
\end{abstract}

Methods and design: The GeliS study is a multicentre cluster-randomized controlled trial. A total number of 2500 pregnant women (singleton pregnancy) with a pre-pregnancy BMI $\geq 18.5 \mathrm{~kg} / \mathrm{m}^{2}$ and $\leq 40 \mathrm{~kg} / \mathrm{m}^{2}$ will be recruited in practices of gynaecologists and midwives in ten Bavarian regions. The intervention comprises three structured and individualised counselling sessions on a healthy diet, regular physical activity as well as weight monitoring during pregnancy and one session after delivery, respectively. The counselling sessions are attached to routine pre- and postnatal visits using standardised materials and procedures. In the control regions, general recommendations for a healthy lifestyle are given. An oral glucose tolerance test is offered to all participants.

The primary outcome is the proportion of participants with excessive GWG. Secondary outcomes include pregnancy and obstetric complications such as frequency of gestational diabetes, preeclampsia and caesarean sections as well as weight retention in the mothers and BMI and other health variables in the offspring. A 5-year follow-up of both mothers and their infants is planned.

Discussion: The GeliS lifestyle intervention programme has been adapted to the existing routine health care system for pregnant women. If shown to be effective, it could be immediately implemented in routine care.

Trial registration: The study protocol is registered at the ClinicalTrials.gov Protocol Registration System (NCT01958307).

Keywords: Lifestyle intervention, Gestational weight gain (GWG), Diet, Exercise, Gestational diabetes, Weight retention, Childhood obesity, Obesity prevention, Pregnancy

\footnotetext{
* Correspondence: hans.hauner@tum.de

${ }^{\dagger}$ Equal contributors

'ZIEL - Research Centre for Nutrition and Food Sciences, Technische

Universität München, Freising-Weihenstephan, Germany

${ }^{3}$ Else Kröner-Fresenius-Centre for Nutritional Medicine, Klinikum rechts der

Isar, Uptown München Campus D, Technische Universität München, Munich,

Germany

Full list of author information is available at the end of the article
} 


\section{Background}

The global prevalence of overweight and obesity has reached an epidemic dimension. In this context, the prevalence of overweight and obesity among women of reproductive age increased substantially [1,2]. Maternal overweight or obesity is associated with a variety of pregnancy and obstetric complications [3-7], such as gestational diabetes mellitus (GDM) [8], preeclampsia [9-11] and an increased risk for macrosomia [12] and caesarean delivery $[13,14]$.

Likewise, recent data suggest that excessive GWG may result in a similar risk profile. Excessive GWG was found to be associated with an increased risk for many adverse maternal and fetal outcomes $[15,16]$ such as large for gestational age (LGA) newborns [17-19] and long-term maternal weight retention after delivery [20-23]. Furthermore, GWG has been reported to be a significant risk factor for childhood obesity [24-33].

To support an optimal pregnancy outcome, the US Institute of Medicine (IOM) provided recommendations for GWG stratified according to BMI of the women before pregnancy [16]. Unfortunately, in many countries including the USA, but also in Germany, an increasing proportion of pregnant women exceed these recommended thresholds $[16,25,34,35]$.

Growing evidence suggests that lifestyle during pregnancy and in the early postnatal period is considerably influencing fetal and infant growth and development. This is an important observation, as pregnancy and the early postnatal period represent critical stages of development for the offspring and may "programme" the risk of metabolic disorders later in life [36,37]. To interfere with this potentially vicious cycle $[38,39]$, adequate intervention strategies which can be introduced into medical practice are urgently required. Some recent studies focussing on a healthy lifestyle during pregnancy and consisting of a balanced diet and regular physical activity showed a reduction in the risk of excessive GWG [40-43].

We recently performed the FeLIPO study (acronym for "Feasibility of a lifestyle intervention in pregnancy to optimize maternal weight development") to assess the potential to prevent excessive GWG by a simple lifestyle intervention [44]. The 2-session intervention programme focussing on a healthy diet, regular physical activity, and self-monitoring of body weight resulted in a significantly lower proportion of women exceeding the IOM recommendations among women in the intervention group (38\%) compared to the control group $(60 \%, \mathrm{p}<0.05)$ [44]. Similar to the results of a recent meta-analysis [45], the FeLIPO study detected a trend for a decreased risk of developing gestational diabetes in the intervention group [44].

Based on the results of this pilot study we are planning to examine the potential of a comprehensive lifestyle programme in pregnant women under "real life" conditions. The GeliS project (acronym for "Gesund leben in der Schwangerschaft"/healthy living in pregnancy) is a public health approach focussing on the prevention of excessive GWG by lifestyle counselling on diet and physical activity during pregnancy, and by selfmonitoring GWG using weight gain charts. The lifestyle intervention programme has been adapted to the German health care system to allow immediate implementation if it is shown to be effective at reducing the rate of excessive GWG and associated risks.

\section{Methods and design \\ Study design and setting}

The GeliS trial is a multicentre and multidisciplinary public health project in ten regions of Bavaria, a federal state of Germany, targeting maternal and fetal health. It is designed as a prospective, cluster-randomized, controlled, open intervention trial. We perform a paired cluster-randomization by matching five pairs of regions according to birth figures, sociodemographic and geographic criteria. Randomization is performed within these pairs and each pair is supervised by an expert centre for nutrition run by the Bavarian State Ministry of Food, Agriculture and Forestry.

Adherence to the study protocol is monitored by the Münchner Studienzentrum at the Technical University of Munich. The Münchner Studienzentrum is furthermore in charge of comprehensive data management according to current standards. The study will be conducted in accordance with the declaration of Helsinki as well as with current local regulatory requirements and laws. Written informed consent will be given by all participants. The study protocol was approved by the ethical committee of the Technical University of Munich and registered at the ClinicalTrials. gov Protocol Registration System (NCT01958307).

\section{Power calculation}

The sample size was calculated based on the proportion of women exceeding weight gain recommendations according to the Institute of Medicine (IOM) guidelines and the results of the FeLIPO study which was conducted as a pilot study [44]. In accordance with data from the Bavarian perinatal register, it is expected that at least $40 \%$ of the women $\left(\mathrm{BMI}>18.5 \mathrm{~kg} / \mathrm{m}^{2}\right)$ will exceed the threshold levels of IOM in the control group (Beyerlein A, November 2009, personal communication on the basis of Bavarian perinatal data from 2007). A decrease to $30 \%$ in the intervention group by adopting the lifestyle intervention programme is assumed. To detect this difference in proportions with $90 \%$ power using a significance level (alpha) of 0.05 and an intraclass correlation coefficient of $0.5 \%$ [46], 1900 pregnant women are needed [47]. To compensate for a possible imbalance in the 
sample sizes between groups as well as a drop-out rate of up to $25 \%$ until delivery, 2500 participants are required for the trial and it is planned to recruit these women in both urban and rural regions reflecting the socioeconomic conditions in the Bavarian population.

\section{Study population \\ Inclusion criteria}

Women aged 18 to 43 years with a singleton pregnancy, a pre-pregnancy BMI $\geq 18.5 \mathrm{~kg} / \mathrm{m}^{2}$ and $\leq 40 \mathrm{~kg} / \mathrm{m}^{2}$, and sufficient German language skills are eligible for the study. These pregnant women are recruited before the $12^{\text {th }}$ week of gestation and written informed consent is mandatory.

\section{Exclusion criteria}

Exclusion criteria are multiple pregnancy, high risk pregnancy prohibiting study participation (contraindications to exercise e.g. placenta praevia, persistent bleeding, cervical incompetence etc.), pre-pregnancy diabetes mellitus or early gestational diabetes, uncontrolled chronic diseases (e.g. thyroid dysfunction), psychiatric or psychosomatic diseases, and any other diseases which could interfere with compliance according to the study protocol.

\section{Drop-out criteria}

Reasons for drop-out are complications during pregnancy as assessed by the trial physician. Women who give birth preterm (delivery before $37^{\text {th }}$ week of gestation) will be excluded from the GWG analysis. These and other reasons for drop-out are carefully documented.

\section{Counsellor recruitment and qualification}

All practice-based gynaecologists including their medical staff as well as all midwives in the selected regions are contacted via information leaflets and by phone. Participating gynaecologists, medical staff or midwives will act as local health care providers and key communicators by implementing counselling sessions for participating pregnant women in the "real-life" setting of routine care. They have to attend a standardised qualifying seminar to ensure competency in lifestyle counselling and standardisation of the intervention. Seminar and information material for the counsellors as well as for the pregnant women were developed in cooperation with the network "Healthy Start - Young Family Network", a project of IN FORM, which is funded by the German Federal Ministry of Food and Agriculture [48].

\section{Participant recruitment}

Before the $12^{\text {th }}$ week of gestation, all pregnant women consecutively presenting in the participating practices are invited by midwives or medical staff to take part in the study. To check inclusion and exclusion criteria and to obtain basic information, participating as well as non- participating women are requested to fill in a short screening questionnaire which includes demographic and anamnestic data relevant to the study. If women fulfill all criteria and are interested to participate, they receive detailed information about the study as well as a form to provide their written informed consent. All these materials were approved by the ethical committee.

\section{The lifestyle intervention programme}

The intervention programme consists of four structured and partially individualised counselling sessions emphasizing diet, physical activity and weight monitoring $\left(12^{\text {th }}-16^{\text {th }}, 16^{\text {th }}-20^{\text {th }}\right.$, and $30^{\text {th }}-34^{\text {th }}$ week of gestation and $6^{\text {th }}-8^{\text {th }}$ week postpartum). The counselling sessions are exclusively given by specifically trained and certified midwives, gynaecologists or medical staff alongside routine prenatal visits and follow a defined curriculum. Pregnant women receive a pedometer and brochures including examples for adequate exercise and a list of local prenatal physical exercise programmes as well as recommendations for a balanced diet in pregnancy according to the above mentioned recommendations on nutrition in pregnancy [49]. Furthermore, they receive a weight gain chart according to their baseline BMI category for self-monitoring of weight development as proposed by the IOM [16]. Figure 1 shows the study scheme including all visits, examinations and other procedures as well as the data that is taken from the pregnant women/mothers and their newborns.

Visit $O\left(\leq 12^{\text {th }}\right.$ week of gestation) represents the screening examination. After checking inclusion and exclusion criteria and obtaining written informed consent, each participant obtains a folder containing questionnaires (on dietary habits and supplements/physical activity/maternal mental health) as well as a pedometer (Beurer $\mathrm{GmbH}$, Ulm, Germany) with adequate instructions.

Visit $1\left(12^{\text {th }}-16^{\text {th }}\right.$ week of gestation): Detailed information about healthy diet and physical activity in pregnancy is given in a face-to-face session of 30 to $45 \mathrm{~min}$. duration. The importance of a healthy lifestyle during pregnancy and its determining factors are explained. Pregnant women learn to handle the principles of healthy eating and to understand the risks of alcohol, smoking and foodborne infections during pregnancy. Further advice relates to GWG, weight monitoring and to critical nutrients in pregnancy (folic acid, iodine, and iron). A list of adequate prenatal physical exercise programmes, brochures including recommendations for a balanced diet in pregnancy as well as a chart for adequate weight gain according to baseline BMI category to self-monitor weight development are handed out. The questionnaires distributed in Visit 0 are collected.

Visit $2\left(16^{\text {th }}-20^{\text {th }}\right.$ week of gestation, 4 weeks distance between the two visits): Specific and detailed individual counselling targeting dietary habits and physical activity also considering the information from the first set of 


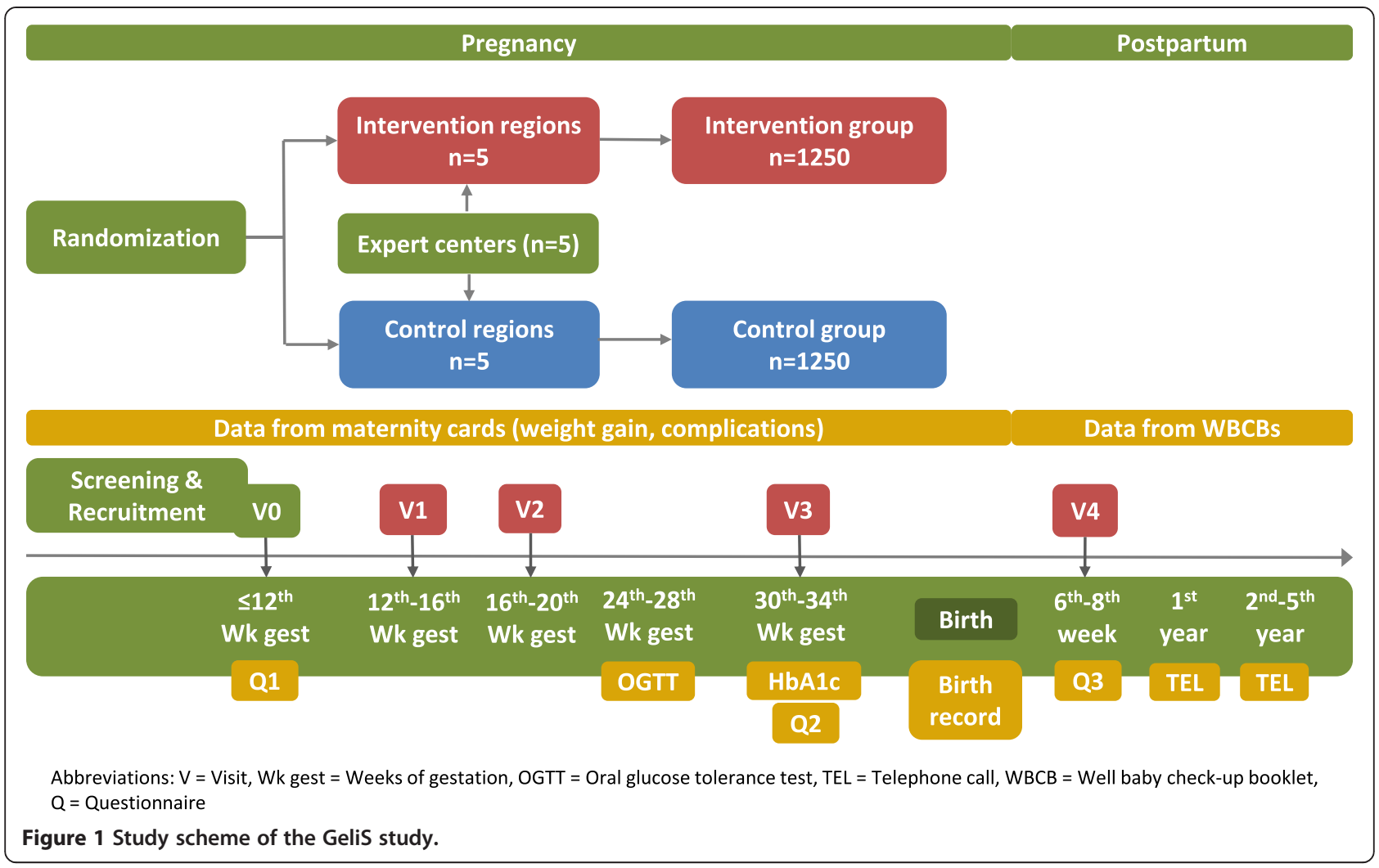

questionnaires is given. Moreover, women are motivated to participate in a standardised two hour oral glucose tolerance test (OGTT) between the $24^{\text {th }}$ and $28^{\text {th }}$ week of gestation, and an appointment for the OGTT is fixed.

Visit $3\left(30^{\text {th }}-34^{\text {th }}\right.$ week of gestation): Repetition and consolidation of contents from the previous visits, with an emphasis on weight monitoring. Counsellors provide information on prenatal maternity courses, post natal exercise classes and on specific problems, such as water retention or back complaints. In addition, the importance of breastfeeding is addressed. A venous EDTA blood sample for measurement of glycosylated haemoglobin (HbA1c) is collected. The second set of questionnaires is handed out.

Visit $4\left(6^{\text {th }}-8^{\text {th }}\right.$ week postpartum): The fourth counselling session includes dietary advice during breastfeeding and information on infant feeding principles. Full breastfeeding is recommended at least up to the age of 4 months. If not returned yet, the second set of questionnaires is collected and the third set of questionnaires (dietary habits and intake of supplements/physical activity/post natal depression/evaluation) is handed out together with a free envelope to return the questionnaires. Maternal weight is measured and documented at each visit.

\section{Control group}

Participants in the control group receive standard prenatal care, including a leaflet about a healthy lifestyle in pregnancy, but no specific individual advice on diet, physical activity or weight gain in pregnancy is given. Data collection and distribution of questionnaires take place within routine prenatal care visits at the same time periods as in the intervention group.

\section{Study outcomes}

\section{Primary outcome}

The primary outcome parameter is the proportion of participating women with excessive GWG as defined by the Institute of Medicine (IOM) [16].

\section{Secondary outcomes}

Secondary outcome parameters include:

- incidence of gestational diabetes $\left(24^{\text {th }}-28^{\text {th }}\right.$ week of gestation, via an oral glucose tolerance test), glycosylated haemoglobin concentration $\left(30^{\text {th }}-34^{\text {th }}\right.$ week of gestation),

- other pregnancy complications such as preeclampsia,

- anthropometric measures and health status of the newborns (birth weight, height, head circumference, LGA, small for gestational age (SGA), APGAR-Score, $\mathrm{pH}$ ),

- obstetric complications (mode of delivery, induction of labour, rate of caesarean sections etc.), 
- maternal dietary and physical activity habits during pregnancy and after delivery,

- maternal body weight after delivery (6-8 weeks postpartum),

- maternal mental health and post natal depression.

\section{Follow-Up}

It is planned to add a follow-up observation programme up to the age of 5 years. Follow-up parameters include breastfeeding and infant feeding practices, growth development and health status of the offspring (including weight and height measurement) as well as maternal weight development.

\section{Outcome measures and data collection Weight measurements, documentation of pregnancy and obstetric complications}

GWG is calculated as the difference between maternal weight at the last prenatal visit and weight at the first visit. The self-reported pre-pregnancy weight is documented at the time of recruitment. At every antenatal visit, weight, blood pressure, urine analysis data as well as pregnancy complications are routinely documented in the maternity card. A standard operation procedure is provided to the participating practices detailing weight measurement. In Germany, maternity cards are delivered to every pregnant woman at their first gynaecological visit as soon as pregnancy is confirmed. To retrieve data regarding complications during pregnancy and delivery and infant anthropometrics, practice staff will copy maternity cards and birth records at the first postnatal visit.

\section{Questionnaires}

\section{Screening-questionnaire}

All women contacted are asked to complete a short anonymous questionnaire containing demographic and anamnestic data independent of study participation.

\section{Dietary assessment}

Dietary habits are recorded twice during pregnancy and once after delivery using a validated Food Frequency Questionnaire (FFQ) according to the DEGS-Study ("German Health Interview and Examination Survey for Adults" conducted by the Robert Koch Institute) [50]. Questions on the intake of dietary supplements are added. Women attending the intervention group receive questionnaires at Visits 0,3 and 4. Women in the control group receive FFQs at the same time points.

\section{Physical activity assessment}

Physical activity is assessed at three time points in both groups (analogous to FFQs) using the validated "Pregnancy physical activity questionnaire" (PPAQ), modestly adjusted to German habits [51]. In addition, a subgroup of women in the intervention group $(\mathrm{n}=250)$ and in the control group $(n=250)$ are asked to carry a pedometer for seven consecutive days. Daily steps are documented in a personal diary which is handed out and completed at the same time points as the FFQ and the PPAQ.

\section{Maternal mental health}

In collaboration with the Mental Health Research Unit of the Helmholtz Zentrum München we have defined a questionnaire with validated elements (WHO-5; PHQ-4; questions from the KORA study - a research platform that is used by national and international research teams [52]). The mental health questionnaire is handed out and collected at the same time points as the FFQ and the PPAQ.

\section{Post natal depression}

Using the Edinburgh Postnatal Depression Scale (EPDSScale) postnatal depression is recorded at 6-8 weeks after delivery. The German version validated by Bergant et al. [53] will be used.

\section{Evaluation}

To assess the acceptance of the lifestyle programme, all participants in the intervention group obtain an evaluation form at the end of the intervention (Visit 4).

\section{OGTT and HbA1c}

To test for gestational diabetes mellitus (GDM), a standardised two-hour oral glucose tolerance test (OGTT) is offered to all participants between the $24^{\text {th }}$ and $28^{\text {th }}$ week of gestation. Tests are performed by the participating gynaecological practices according to the 2011 guidelines of the German Diabetes Society (DDG) and the German Society of Gynaecology and Obstetrics (DGGG) [54] which is in line with the guidelines of the International Association of the Diabetes and Pregnancy Study Groups (IADPSG) [55]. After an overnight fast, serum glucose level is measured before, one and two hours after intake of 75 grams glucose (Dextro ${ }^{\circ} \mathrm{OGT}$, Roche, Mannheim). Treatment will be performed at the discretion of the participating gynaecologist. Every participating gynaecological practice will receive information about the DDG/DGGG guidelines for the management of gestational diabetes [54] and is asked to consider these principles as soon as GDM is diagnosed.

To obtain additional information on the presence and severity of diagnosed GDM, EDTA blood is collected for the measurement of the glycosylated haemoglobin concentration between the $30^{\text {th }}$ and $34^{\text {th }}$ week of gestation. 


\section{Follow-Up - well baby check-up booklet}

Follow-up interviews (by phone call or e-mail, upon preference) will be arranged after one, and annually up to 5 years after delivery to record maternal body weight as well as infant anthropometrics. The infant anthropometrics are based on measured data from the well baby check-up booklet provided to all mothers in Germany at birth to promote regular health examinations by the family pediatricians. This is part of the free health check-up programme for infants in the German health care system. The (preschool-) programme comprises standardised health examinations at six time points during the first year of age (immediately after delivery, $3^{\text {th }}$ $10^{\text {th }}$ day of age, $4^{\text {th }}-5^{\text {th }}$ week of age, $3^{\text {th }}-4^{\text {th }}$ month of age, $6^{\text {th }}-7^{\text {th }}$ month of age, $10^{\text {th }}-12^{\text {th }}$ month of age) as well as annually up to the age of five $\left(21^{\text {th }}-24^{\text {th }}\right.$ month of age, $34^{\text {th }}-36^{\text {th }}$ month of age, $46^{\text {th }}-48^{\text {th }}$ month of age, $60^{\text {th }}-64^{\text {th }}$ month of age). In Bavaria, the programme is currently utilized by approximately $84.5 \%$ of all parents [56]. All mothers are explicitly encouraged to participate in this programme. In addition, breastfeeding and infant feeding practices are assessed and documented. All data are pseudonymised before they are transferred to data management at the Münchner Studienzentrum for database entry.

\section{Discussion}

Excessive GWG is well known to be associated with a higher risk of pregnancy and birth complications [15-19,57,58]. Moreover, excessive GWG has also been reported to favour maternal weight retention [23] and to promote childhood obesity [24-33]. According to recent surveys $[16,59,60]$, an increasing number of pregnant women show excessive GWG as defined by the guideline of the Institute of Medicine [16]. Therefore, there is an urgent demand for effective strategies to stop this trend in order to avoid its potential adverse consequences for both mother and child.

We recently published the results of a feasibility study performed in 8 gynaecological practices in the Munich area using a cluster-randomized design. Two sessions of lifestyle counselling were sufficient to achieve a significant reduction in the percentage of women with excessive GWG compared to a control group. There was also less weight retention in the mothers at month 4 as well as a tendency for a lower rate of gestational diabetes and less caesarean sections [44]. These results are in line with a number of other small intervention studies aiming to limit GWG during pregnancy. Most studies showed a moderate reduction in GWG, but were not adequately powered for other outcome variables such as GDM or obstetric complications [40-43,61-64].

The present study is based on this experience, but aims to explore this concept of lifestyle intervention in the routine health care system. In Germany, regular preand postnatal care is well established and utilised by almost every pregnant woman. However, the current health care system for pregnant women and their offspring is solely focussing on obstetrical aspects and, after birth, on the functional development of the children. Our concept is to complement this system by a lifestyle programme combining a healthy diet, regular physical activity, and self-monitoring of body weight to avoid excessive weight gain and its potential risks for both mother and child.

The increased risk of long-term obesity in the offspring of mothers with excessive GWG [24,29,30,33,57] is hypothesized to result from a malprogramming process during pregnancy and early postnatal life $[3,65]$. This concept of fetal programming is attractive, but since the current evidence is mainly based on animal data and retrospective human association studies it has not yet been proven for humans. Therefore, a specific feature of our trial is to look at weight development in the children up to the age of 5 years utilising the unique health check-up system for children in Germany. Thus, this study may also serve to examine the hypothesis that a healthy lifestyle during pregnancy can reduce the risk of early childhood obesity in the offspring.

Pregnancy and lactation is not only a critical period for long-term malprogramming, but can also be considered as a "teachable moment" describing a life situation that is thought to motivate women to adopt a risk-reducing health behaviour [42]. As already seen in our feasibility study, the compliance with a lifestyle programme may be particularly high in this phase. In the interest of the health of their babies, women are more responsive to lifestyle counselling than at any other time. In addition, if the concept of fetal programming turns out to be valid, a temporarily limited lifestyle change may not only gain more acceptance by women, but could have a sustained beneficial effect.

In addition to the long-term follow-up of the infants, another potential strength of the GeliS study is the "real world" setting. The study is performed within the framework of the German healthcare system and represents a true public health approach to this upcoming health challenge. The intervention programme is paid for by the largest regional statutory health insurance and it is planned to implement the lifestyle intervention programme into routine care if a benefit for both mother and child can be demonstrated. An accompanying health economic analysis is planned to study the cost-effectiveness of the intervention.

A weakness of the study may be that there are other potentially relevant perinatal factors which may modify the obesity risk in this early phase of life. In a recent analysis of birth cohort data pre-pregnancy BMI was identified as the most powerful determinant of childhood obesity [66]. 
However, a recent longitudinal study in Southampton, UK showed that only a small proportion of women followed nutrition and lifestyle recommendations pre-pregnancy, but this proportion increased during pregnancy [67]. Thus, prevention of excessive GWG together with the other recommendations for a healthy lifestyle, e.g. stopping smoking and alcohol consumption, may be the most promising and practicable strategy to address the lifestyleassociated health issues previously discussed.

In summary, the GeliS trial will examine the potential of a strategy implemented to avoid excessive GWG and, thus, to prevent the GWG-associated health risks for both mother and child in the routine health care of pregnant women. If shown to be effective, it is important to note that the collaborating statutory health insurance fund plans to implement the lifestyle counselling into the routine care programme for pregnant women. Then, promotion of a healthy lifestyle including management of weight gain during pregnancy could become an easy and valuable strategy for the primary prevention of adverse consequences of excessive GWG in both women and their children.

\section{Competing interests}

The authors declare that they have no competing interests.

\section{Authors' contributions}

$H H, K R, J K, E R$ and LK are members of the Gelis study group and contributed to the design of the study. $\mathrm{HH}$ and $\mathrm{KR}$ developed the study protocol. $\mathrm{HH}, \mathrm{KR}$, ER and LK established the lifestyle intervention programme. KU provided advice on power calculation and statistical analyses. JK, KR, and $\mathrm{HH}$ wrote the manuscript. All authors read and approved the final manuscript.

\section{Acknowledgements}

The study is funded by the Else Kröner-Fresenius Centre for Nutritional Medicine at the Technische Universität München, the Competence Centre for Nutrition (KErn) in Bavaria, the Bavarian State Ministry of Food, Agriculture and Forestry, the Bavarian State Ministry of Health and Care (Health Initiative "Gesund.Leben.Bayern."), as well as AOK Bayern, the largest statuatory health insurance in Bavaria. We gratefully acknowledge the valuable contribution from the network "Healthy Start - Young Family Network", a project of IN FORM (Maria Flothkötter, Katharina Krüger, Julia Bonfig), funded by the German Federal Ministry of Food and Agriculture, Bonn, and the Münchner Studienzentrum at Technische Universität München (Dr. Alfred Zollner, Dr. Elisabeth André, Caspar Sennefelder). Project managers at the expert centres for nutrition/community catering at the regional offices (AELF) of the Bavarian State Ministry of Food, Agriculture and Forestry (StMELF) are coordinating the study in the ten study regions. Finally, the input from Dr. Uta Engels, Sports Centre, University of Regensburg, Prof. Karl-Heinz Ladwig, Institute of Epidemiology, Helmholtz Zentrum München, Prof. K.T.M. Schneider, Division of Obstetrics and Perinatal Medicine, Technische Universität München, and Gabi Pfeifer, sports scientist at KErn, is gratefully acknowledged. We are also indebted to the Board of Trustees: Maria Flothkötter, aid infodienst Ernährung, Landwirtschaft, Verbraucherschutz e.V., Bonn; Dr. Beatrix Heilig, Bavarian State Ministry of Health and Care; Marion Kratzmair, Bavarian State Ministry of Food, Agriculture and Forestry; Dr. Wolfram Schaecke, Competence Centre for Nutrition, Freising/Kulmbach, and Dr. Annette Scheder, AOK Bayern. We would also like to thank the Bayerische Landesärztekammer, Bayerischer Hebammen Landesverband e.V. and the Company Beurer GmbH, Ulm, for supporting activities as well as Dr. Lynne Cresswell for critical reading of the manuscript. This work was supported by the German Research Foundation (DFG) and the Technische Universität München within the funding programme Open Access Publishing.

\section{Author details}

${ }^{1}$ ZIEL - Research Centre for Nutrition and Food Sciences, Technische Universität München, Freising-Weihenstephan, Germany. ${ }^{2}$ Competence Centre for Nutrition (KErn), Freising, Germany. ${ }^{3}$ Else Kröner-Fresenius-Centre for Nutritional Medicine, Klinikum rechts der Isar, Uptown München Campus D, Technische Universität München, Munich, Germany. ${ }^{4}$ Department of Medical Statistics and Epidemiology, Technische Universität München, Munich, Germany.

Received: 28 February 2014 Accepted: 20 March 2014

Published: 28 March 2014

\section{References}

1. Swinburn BA, Sacks G, Hall KD, McPherson K, Finegood DT, Moodie ML, Gortmaker SL: The global obesity pandemic: shaped by global drivers and local environments. Lancet 2011, 378:804-814.

2. World Health Organization: WHO | Obesity and overweight. http://www. who.int/mediacentre/factsheets/fs311/en/index.html.

3. Guelinckx I, Devlieger R, Beckers K, Vansant G: Maternal obesity: pregnancy complications, gestational weight gain and nutrition. Obes Rev 2008, 9:140-150.

4. Nelson SM, Matthews P, Poston L: Maternal metabolism and obesity: modifiable determinants of pregnancy outcome. Hum Reprod Update 2010, 16:255-275.

5. Davies GA, Gregory AL, Maxwell C, McLeod L, Gagnon R, Basso M, Bos H, Delisle M, Farine D, Hudon L, Menticoglou S, Mundle W, Murphy-Kaulbeck L, Ouellet A, Pressey T, Roggensack A, Leduc D, Ballerman C, Biringer A, Duperron L, Jones D, Lee LS, Shepherd D, Wilson K: SOGC Clinical Practice Guidelines: Obesity in pregnancy. No. 239, February 2010. Int J Gynaecol Obstet 2010, 110:167-173.

6. Adamo KB, Ferraro ZM, Brett KE: Can we modify the intrauterine environment to halt the intergenerational cycle of obesity? Int J Environ Res Public Health 2012, 9:1263-1307.

7. Catalano PM, Ehrenberg HM: The short- and long-term implications of maternal obesity on the mother and her offspring. BJOG 2006, 113:1126-1133.

8. Torloni MR, Betrán AP, Horta BL, Nakamura MU, Atallah AN, Moron AF, Valente O: Prepregnancy BMI and the risk of gestational diabetes: a systematic review of the literature with meta-analysis. Obes Rev 2009, 10:194-203

9. Tsoi E, Shaikh H, Robinson S, Teoh TG: Obesity in pregnancy: a major healthcare issue. Postgrad Med J 2010, 86:617-623.

10. Leeners B, Rath W, Kuse S, Irawan C, Imthurn B, Neumaier-Wagner P: BMI: new aspects of a classical risk factor for hypertensive disorders in pregnancy. Clin Sci 2006, 111:81-86.

11. Sebire NJ, Jolly M, Harris JP, Wadsworth J, Joffe M, Beard RW, Regan L, Robinson S: Maternal obesity and pregnancy outcome: a study of 287,213 pregnancies in London. Int J Obes Relat Metab Disord 2001, 25:1175-1182.

12. Ehrenberg HM, Mercer BM, Catalano PM: The influence of obesity and diabetes on the prevalence of macrosomia. Am J Obstet Gynecol 2004, 191:964-968.

13. Poobalan AS, Aucott LS, Gurung T, Smith WCS, Bhattacharya S: Obesity as an independent risk factor for elective and emergency caesarean delivery in nulliparous women-systematic review and meta-analysis of cohort studies. Obes Rev 2009, 10:28-35.

14. Heslehurst N, Simpson H, Ells LJ, Rankin J, Wilkinson J, Lang R, Brown TJ, Summerbell CD: The impact of maternal BMI status on pregnancy outcomes with immediate short-term obstetric resource implications: a meta-analysis. Obes Rev 2008, 9:635-683.

15. Rasmussen KM, Catalano PM, Yaktine AL: New guidelines for weight gain during pregnancy: what obstetrician/gynecologists should know. Curr Opin Obstet Gynecol 2009, 21:521-526.

16. Rasmussen KM, Yaktine AL: Weight gain during pregnancy: Reexamining the guidelines. Washington, DC: National Academies Press; 2009.

17. Liu Y, Dai W, Dai X, Li Z: Prepregnancy body mass index and gestational weight gain with the outcome of pregnancy: a 13-year study of 292,568 cases in China. Arch Gynecol Obstet 2012, 286:905-911.

18. Ferraro ZM, Barrowman N, Prud'homme D, Walker M, Wen SW, Rodger M, Adamo KB: Excessive gestational weight gain predicts large for 
gestational age neonates independent of maternal body mass index. J Matern Fetal Neonatal Med 2012, 25:538-542.

19. DeVader SR, Neeley HL, Myles TD, Leet TL: Evaluation of gestational weight gain guidelines for women with normal prepregnancy body mass index. Obstet Gynecol 2007, 110:745-751.

20. Linné $Y$, Dye $L$, Barkeling $B$, Rössner $S$ : Long-term weight development in women: a 15-year follow-up of the effects of pregnancy. Obes Res 2004, 12:1166-1178.

21. Rode L, Kjærgaard H, Ottesen B, Damm P, Hegaard HK: Association between gestational weight gain according to body mass index and postpartum weight in a large cohort of Danish women. Matern Child Health J 2012, 16:406-413.

22. Begum F, Colman I, McCargar LJ, Bell RC: Gestational weight gain and early postpartum weight retention in a prospective cohort of alberta women. J Obstet Gynaecol Can 2012, 34:637-647.

23. Nehring I, Schmoll S, Beyerlein A, Hauner H, Kries Von R: Gestational weight gain and long-term postpartum weight retention: a meta-analysis. Am J Clin Nutr 2011, 94:1225-1231.

24. Olson CM, Strawderman MS, Dennison BA: Maternal weight gain during pregnancy and child weight at age 3 years. Matern Child Health J 2009, 13:839-846.

25. von Kries R, Ensenauer R, Beyerlein A, Amann-Gassner U, Hauner H, Rosario AS: Gestational weight gain and overweight in children: Results from the cross-sectional German KiGGS study. Int J Pediatr Obes 2011, 6:45-52.

26. Margerison-Zilko CE, Shrimali BP, Eskenazi B, Lahiff M, Lindquist AR, Abrams BF: Trimester of maternal gestational weight gain and offspring body weight at birth and age five. Matern Child Health J 2012, 16:1215-1223.

27. Beyerlein A, Nehring I, Rzehak P, Heinrich J, Müller MJ, Plachta-Danielzik S, Wabitsch M, Weck M, Brenner H, Rothenbacher D, Kries Von R: Gestational weight gain and body mass index in children: results from three german cohort studies. PLOS ONE 2012, 7:e33205.

28. Ensenauer R, Chmitorz A, Riedel C, Fenske N, Hauner H, Nennstiel-Ratzel U, Kries Von R: Effects of suboptimal or excessive gestational weight gain on childhood overweight and abdominal adiposity: results from a retrospective cohort study. Int J Obes (Lond) 2013, 37:505-512.

29. Moreira P, Padez C, Mourão-Carvalhal I, Rosado V: Maternal weight gain during pregnancy and overweight in Portuguese children. Int J Obes (Lond) 2007, 31:608-614.

30. Oken E, Taveras EM, Kleinman KP, Rich-Edwards JW, Gillman MW: Gestational weight gain and child adiposity at age 3 years. Am J Obstet Gynecol 2007, 196(322):e1-e8

31. Reynolds RM, Osmond C, Phillips DIW, Godfrey KM: Maternal BMI, parity, and pregnancy weight gain: influences on offspring adiposity in young adulthood. J Clin Endocrinol Metab 2010, 95:5365-5369.

32. Schack-Nielsen L, Michaelsen KF, Gamborg M, Mortensen EL, Sørensen TIA: Gestational weight gain in relation to offspring body mass index and obesity from infancy through adulthood. Int J Obes (Lond) 2010, 34:67-74.

33. Wrotniak BH, Shults J, Butts S, Stettler N: Gestational weight gain and risk of overweight in the offspring at age $7 \mathrm{y}$ in a multicenter, multiethnic cohort study. Am J Clin Nutr 2008, 87:1818-1824.

34. Bergmann KE, Bergmann RL, Ellert U, Dudenhausen JW: Perinatale Einflussfaktoren auf die spätere Gesundheit. Ergebnisse des Kinder- und Jugendgesundheitssurveys (KiGGS). Bundesgesundheitsblatt Gesundheitsforschung Gesundheitsschutz 2007, 50:670-676.

35. Schiessl B, Beyerlein A, Lack N, Kries Von R: Temporal trends in pregnancy weight gain and birth weight in Bavaria 2000-2007: slightly decreasing birth weight with increasing weight gain in pregnancy. J Perinat Med 2009, 37:374-379.

36. Taylor PD, Poston L: Developmental programming of obesity in mammals. Exp Physiol 2007, 92:287-298.

37. Battista M, Hivert M, Duval K, Baillargeon J: Intergenerational cycle of obesity and diabetes: how can we reduce the burdens of these conditions on the health of future generations? Exp Diabetes Res 2011, 2011:596060.

38. Catalano PM: Obesity and pregnancy-the propagation of a viscous cycle? J Clin Endocrinol Metab 2003, 88:3505-3506.

39. Dabelea D, Crume T: Maternal environment and the transgenerational cycle of obesity and diabetes. Diabetes 2011, 60:1849-1855.

40. Streuling I, Beyerlein A, Kries Von R: Can gestational weight gain be modified by increasing physical activity and diet counseling? A meta-analysis of interventional trials. Am J Clin Nutr 2010, 92:678-687.
41. Wolff S, Legarth J, Vangsgaard K, Toubro S, Astrup A: A randomized trial of the effects of dietary counseling on gestational weight gain and glucose metabolism in obese pregnant women. Int J Obes (Lond) 2008, 32:495-501.

42. Phelan S, Phipps MG, Abrams B, Darroch F, Schaffner A, Wing RR: Randomized trial of a behavioral intervention to prevent excessive gestational weight gain: the Fit for Delivery Study. Am J Clin Nutr 2011, 93:772-779.

43. Vinter CA, Jensen DM, Ovesen P, Beck-Nielsen H, Jørgensen JS: The LiP (Lifestyle in Pregnancy) study: a randomized controlled trial of lifestyle intervention in 360 obese pregnant women. Diabetes Care 2011 34:2502-2507

44. Rauh K, Gabriel E, Kerschbaum E, Schuster T, Von KR, Amann-Gassner U, Hauner $\mathrm{H}$ : Safety and efficacy of a lifestyle intervention for pregnant women to prevent excessive maternal weight gain: a clusterrandomized controlled trial. BMC Pregnancy Childbirth 2013, 13:151

45. Oteng-Ntim E, Varma R, Croker H, Poston L, Doyle P: Lifestyle interventions for overweight and obese pregnant women to improve pregnancy outcome: systematic review and meta-analysis. BMC Med 2012, 10:47.

46. Hannan PJ, Murray DM, Jacobs DR, McGovern PG: Parameters to aid in the design and analysis of community trials: intraclass correlations from the Minnesota Heart Health Program. Epidemiology 1994, 5:88-95.

47. Donner A, Klar N: Design and analysis of cluster randomization trials in health research. London: Arnold Publishing Company; 2000.

48. Gesund ins Leben - Netzwerk Junge Familie. http://www.gesund-ins-leben.de/

49. Koletzko B, Bauer CP, Bung P, Cremer M, Flothkötter M, Hellmers C, Kersting M, Krawinkel M, Przyrembel H, Rasenack R, Schäfer T, Vetter K, Wahn U, Weißenborn A, Wöckel A: Nutrition in pregnancy. Practice recommendations of the Network "Healthy Start - Young Family Network". Dtsch Med Wochenschr 2012, 137(25-26):1366-1372.

50. Haftenberger M, Heuer T, Heidemann C, Kube F, Krems C, Mensink Gert BM: Relative validation of a food frequency questionnaire for national health and nutrition monitoring. Nutr J 2010, 9:36.

51. Chasan-Taber L, Schmidt MD, Roberts DE, Hosmer D, Markenson G, Freedson PS: Development and validation of a Pregnancy Physical Activity Questionnaire. Med Sci Sports Exerc 2004, 36:1750-1760.

52. Helmholtz Zentrum München Deutsches Forschungszentrum für Gesundheit und Umwelt (GmbH): KORA - Kooperative Gesundheitsforschung in der Region Augsburg: KORA Startseite. http://www.helmholtz-muenchen.de/kora.

53. Bergant AM, Nguyen T, Heim K, Ulmer H, Dapunt O: Deutschsprachige Fassung und Validierung der "Edinburgh postnatal depression scale". Dtsch Med Wochenschr 1998, 123:35-40.

54. Kleinwechter H, Schäfer-Graf U, Bührer C, Hoesli I, Kainer F, Kautzky-Willer A, Pawlowski B, Schunk K, Somville T, Sorger M: Gestationsdiabetes mellitus (GDM) - Evidenzbasierte Leitlinie zur Diagnostik, Therapie u. Nachsorge der Deutschen Diabetes-Geseelschaft (DDG) und der Deutschen Gesellschaft für Gynäkologie und Geburtshilfe (DGGG). 2011. www.dggg.de/fileadmin/ public docs/Dokumente/Leitlinien/3-3-3-Gestationsdiabetes-2011.pdf.

55. Metzger BE, Gabbe SG, Persson B, Buchanan TA, Catalano PA, Damm P, Dyer AR, Leiva A, Hod M, Kitzmiler JL, Lowe LP, Mclntyre HD, Oats Jeremy JN, Omori Y, Schmidt Ml: International association of diabetes and pregnancy study groups recommendations on the diagnosis and classification of hyperglycemia in pregnancy. Diabetes Care 2010, 33:676-682.

56. Schulz R, Hölscher G, Schneider T, Hachmeister A, Scholz S, Nennstiel-Ratzel $\mathrm{U}$ : Gesundheit der Vorschulkinder in Bayern: Ergebnisse der Schuleingangsuntersuchung zum Schuljahr 2008/2009, Statistisch-epidemiologischer Bericht. Bayerisches Landesamt für Gesundheit und Lebensmittelsicherheit: Band 4 der Schriftenreihe Schuleingangsuntersuchung in Bayern; 2013.

57. Margerison Z, Claire E, Rehkopf D, Abrams B: Association of maternal gestational weight gain with short- and long-term maternal and child health outcomes. Am J Obstet Gynecol 2010, 202(574):e1-e8.

58. Durie DE, Thornburg LL, Glantz JC: Effect of second-trimester and thirdtrimester rate of gestational weight gain on maternal and neonatal outcomes. Obstet Gynecol 2011, 118:569-575.

59. Chu SY, Callaghan WM, Bish CL, D'Angelo D: Gestational weight gain by body mass index among US women delivering live births, 2004-2005: fueling future obesity. Am J Obstet Gynecol 2009, 200(271):e1-e7.

60. Lowell H, Miller DC: Weight gain during pregnancy: adherence to Health Canada's guidelines. Health Rep 2010, 21:31-36.

61. Thornton YS, Smarkola C, Kopacz SM, Ishoof SB: Perinatal outcomes in nutritionally monitored obese pregnant women: a randomized clinical trial. J Natl Med Assoc 2009, 101:569-577. 
62. Quinlivan JA, Lam LT, Fisher J: A randomised trial of a four-step multidisciplinary approach to the antenatal care of obese pregnant women. Aust N Z J Obstet Gynaecol 2011, 51:141-146.

63. Luoto R, Kinnunen TI, Aittasalo M, Kolu P, Raitanen J, Ojala K, Mansikkamäki K, Lamberg S, Vasankari T, Komulainen T, Tulokas S: Primary prevention of gestational diabetes mellitus and large-for-gestational-age newborns by lifestyle counseling: a cluster-randomized controlled trial. PLOS Med 2011, 8:e1001036.

64. Ruchat S, Davenport MH, Giroux I, Hillier M, Batada A, Sopper MM, Hammond JA, Joanne MS, Mottola MF: Nutrition and exercise reduce excessive weight gain in normal-weight pregnant women. Med Sci Sports Exerc 2012, 44:1419-1426.

65. Alfaradhi MZ, Ozanne SE: Developmental programming in response to maternal overnutrition. Front Genet 2011, 2:27.

66. Catalano PM, Farrell K, Thomas A, Huston-Presley L, Mencin P, De M, Sylvie $H$, Amini SB: Perinatal risk factors for childhood obesity and metabolic dysregulation. Am J Clin Nutr 2009, 90:1303-1313.

67. Crozier SR, Robinson SM, Borland SE, Godfrey KM, Cooper C, Inskip HM: Do women change their health behaviours in pregnancy? Findings from the Southampton Women's Survey. Paediatr Perinat Epidemiol 2009, 23:446-453.

doi:10.1186/1471-2393-14-119

Cite this article as: Rauh et al:: Healthy living in pregnancy: a clusterrandomized controlled trial to prevent excessive gestational weight gain - rationale and design of the GeliS study. BMC Pregnancy and Childbirth 2014 14:119.

\section{Submit your next manuscript to BioMed Central and take full advantage of:}

- Convenient online submission

- Thorough peer review

- No space constraints or color figure charges

- Immediate publication on acceptance

- Inclusion in PubMed, CAS, Scopus and Google Scholar

- Research which is freely available for redistribution 